$R M x A C, \mathbf{5 3}, 206-214(2021)$

(c) 2021: Instituto de Astronomía, Universidad Nacional Autónoma de México

https://doi.org/10.22201/ia.14052059p.2021.53.40

\title{
LONG-TERM PHOTOMETRY WITH SKYNET ROBOTIC TELESCOPE NETWORK
}

\author{
S. Zola ${ }^{1,2}$, V. Kouprianov ${ }^{3}$, D. E. Reichart ${ }^{3}$, G. Bhatta ${ }^{1}$, and D. B. Caton ${ }^{4}$
}

\section{RESUMEN}

En este artículo, describimos la red de telescopios robóticos Skynet, una red de aproximadamente 2 docenas de pequeños telescopios ópticos que abarcan cuatro continentes. Para un posible usuario de la red, proporcionamos detalles sobre su funcionamiento. y damos ciertas advertencias que pueden afectar las observaciones tomadas bajo el control del software Skynet. El rendimiento real de la red se demuestra mediante observaciones a largo plazo de dos blázares: DO 49 y 3C 279. Mostramos sus curvas de luz fotométricas adquiridas con un subconjunto de telescopios Skynet en la observación realizada en el bienio 2018/19 en diversas estaciones. Se proporcionan resultados preliminares del análisis de la variabilidad de estos dos objetivos, incluidas las propiedades cuantitativas de las variaciones de luz y los períodos de posibles oscilaciones cuasiperiódicas (QPOs) que se encuentran en las curvas de luz estacionales.

\section{ABSTRACT}

In this paper, we describe the Skynet Robotic Telescope Network, a network of about 2 dozen small optical telescopes spanning four continents. For a prospective user of the network, we provide details on its operation and mention certain caveats potentially affecting observations taken under the control of Skynet software. The actual performance of the network is demonstrated by long-term observations of two blazars: OJ 49 and 3C 279. We show their photometric light curves acquired with a subset of Skynet telescopes in the 2018/19 observing seasons. Preliminary results of the analysis of the variability of these two targets are given, including the quantitative properties of light variations and the periods of possible Quasi Periodic Oscillations (QPOs) found in the seasonal light curves.

Key Words: Telescopes — galaxies: active — galaxies: photometry — BL Lacertae objects: individual: OJ 49, 3C 279

\section{OBSERVING WITH SKYNET TELESCOPES}

\subsection{Skynet at a glance}

Skynet Robotic Telescope Network was started by D. Reichart at the University of North Carolina at Chapel Hill (UNC) in 2004 as an effort to build a network of telescopes with a primary goal of observing gamma-ray burst (GRB) afterglows. Currently it comprises around 20 small (0.4-1 meter aperture) optical telescopes in the Americas, Australia, and Europe and a 20-meter radio telescope at Green Bank Observatory in West Virginia (USA). Its core is a set of dedicated PROMPT (Panchromatic Robotic Optical Monitoring and Polarimetry Telescope) telescopes owned by UNC and installed in

\footnotetext{
${ }^{1}$ Astronomical Observatory, Jagiellonian University, ul. Orla 171, 30-244 Krakow, Poland (szola@oa.uj.edu.pl).

${ }^{2}$ Mt. Suhora Observatory, Pedagogical University, ul. Podchorazych 2, 30-084 Krakow, Poland.

${ }^{3}$ Department of Physics and Astronomy, University of North Carolina at Chapel Hill, 120 E. Cameron Ave., Phillips Hall, CB No3255, Chapel Hill, NC 27599, USA.

${ }^{4}$ Dept. of Physics and Astronomy, Appalachian State University, 231 Garwood Hall, 525 Rivers St., ASU Box 32106, Boone, NC 28608, USA.
}

Chile $\left(3 \times 16^{\prime \prime}\right.$ telescopes $+3 \times 24^{\prime \prime}+32^{\prime \prime}+40^{\prime \prime}, 60^{\prime \prime}$ proposed, Polarimeter and spectrograph in development), Australia $\left(4 \times 17^{\prime \prime}\right.$, proposed $17^{\prime \prime}$ or $\left.40^{\prime \prime}\right)$, and Canada $\left(16^{\prime \prime}\right)$. Apart from them, Skynet includes a number of partnering university telescopes, which number is constantly growing. Skynet is funded mostly by the National Science Foundation (NSF), but also by NASA and Mt. Cuba Astronomical Foundation.

About a half of Skynet activities is dedicated to research. Its main subjects of interest cover many hot topics of modern astronomy and include GRBs, gravitational wave sources, Fast Radio Bursts (FRBs), blazars (both optical and radio), supernovae, supernova remnants in radio, novae, pulsating white dwarfs and hot subdwarfs, variable stars, eclipsing binaries, exoplanetary systems, trans-Neptunian objects (TNOs) and Centaurs, asteroids, and near-Earth objects (NEOs). Skynet is the leading NEO tracker in southern hemisphere. Publications involving Skynet appear every 20 days and its results were published 5 times in Nature and Science so far. Another half of Skynet telescope time 

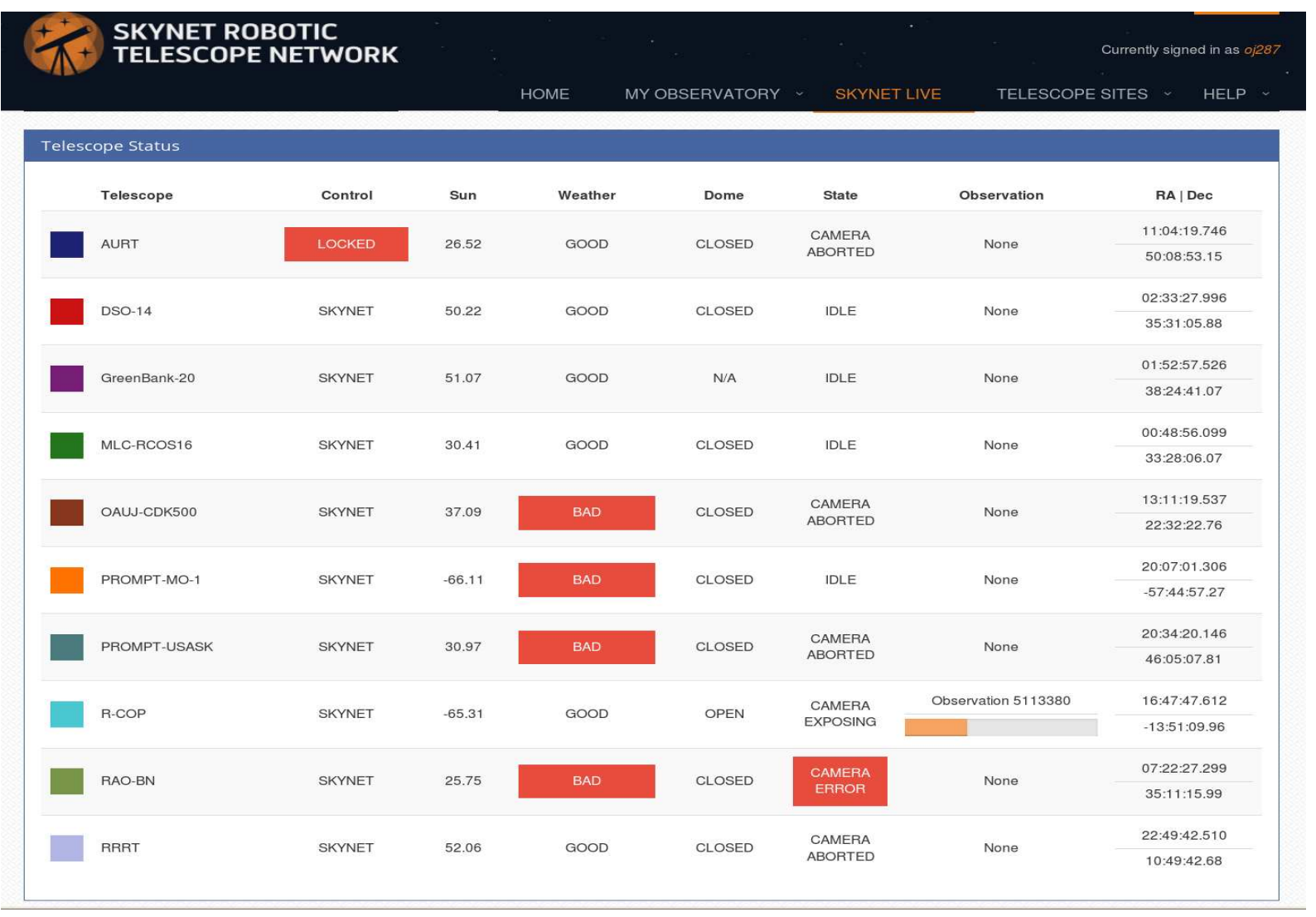

Fig. 1. Skynet Live page. For privacy reasons, the job details are visible only to its owner, their supervisor, if any, and to the telescope admins.

is spent on education and public engagement. Up to now, Skynet have served around 50000 students of all ages and participated in three large NSF programs on education.

The current Skynet telescope control software Terminator is based on ASCOM and works with most commercially available hardware (telescopes, domes, cameras, filter wheels, and focusers). Under development is a next-generation telescope control system (TCS) aimed at a greater flexibility and support of large telescopes and complex instrumentation.

Once the observation is submitted, the user can watch its progress, preview individual images taken as JPEG or PNG, and download the Skynet is open for collaboration. To participate, a potential partner must own a $40 \mathrm{~cm}$ class or bigger robotic telescope operated by ASCOM-compliant software, with a weather station, a computerized dome, and a reliable internet connection, fully ready for remote control. Knowledgeable and dedicated local support staff is also in a great need to facilitate uninterrupted operation. For every member telescope, Skynet expects up to $10 \%$ of observing time to go to its education and science programs. In return, the member takes advantage of Skynet web interface and API (see next section), dynamic scheduler, account management infrastructure, and image analysis interface. Furthermore, Skynet telescope owner can allocate their observing time to anyone or trade it with other telescope owners, thus gaining access to the resources of the whole network.

\subsection{User Interface}

Skynet provides its facilities to registered users individuals, students, and members of research teams and collaborations. Observers interact with Skynet by means of the web user interface (UI) at https://skynet.unc.edu. Its main purpose is to allow the users to submit observations, monitor their progress, and preview and download the images and calibration data. Apart from that, the UI provides the ability for telescope, site, group, and collaboration administrators to watch and control the state of telescopes, their parameters and performance, prior- 


$$
\text { Telescope Status }
$$

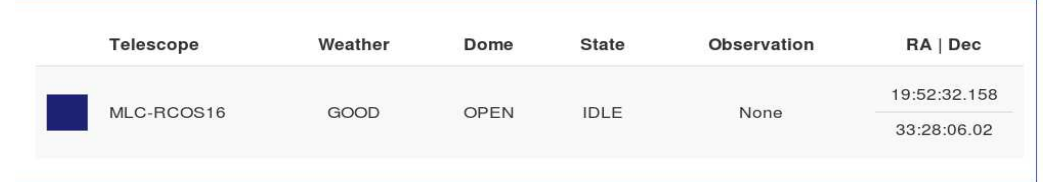

Live Site View

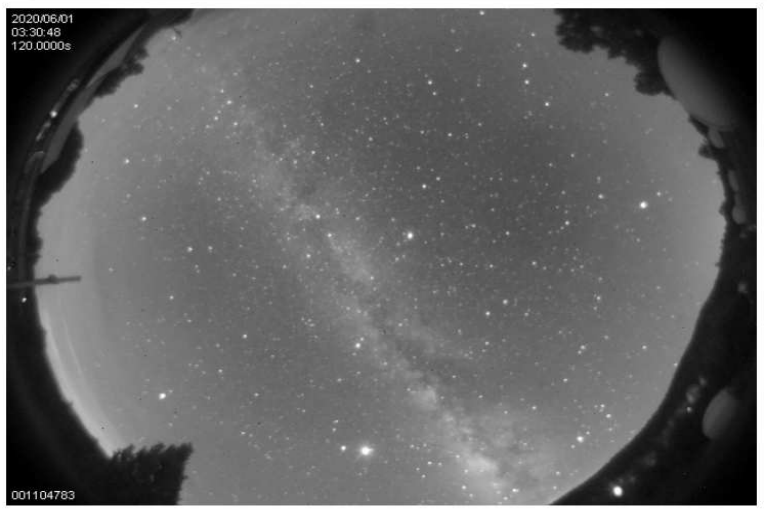

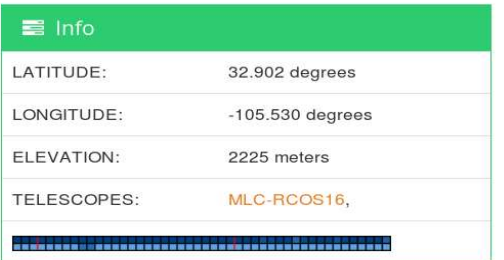
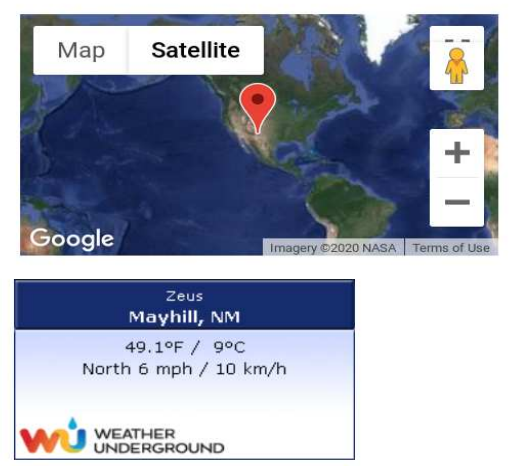

Recent Images

Fig. 2. Information on sites and telescopes. The quality of sky conditions can be seen in the observatory all-sky camera image (if available).

itize the allocation of time slots, control group and collaboration membership, and perform other administrative tasks. Skynet Live page (Fig. 1.1) shows a summary of all telescopes currently connected to Skynet, indicating the current telescope's operation mode, weather conditions, and the job that it is executing.

\subsection{Summary of the Advantages and Pitfalls of Observing with Skynet}

The Telescope Sites drop-down menu (Fig. 1.1) can be used to view the information on the individual Skynet sites and telescopes, including their location, characteristics of instrumentation, webcam footage (if available), ClearSky charts (https:// www.cleardarksky.com/csk/), and recently taken images.

From the observer's perspective, the most important features of the Skynet UI are submitting observations and getting data back when they are completed. The website UI provides a convenient and flexible way of defining an observation, what- ever simple or complex it is. The user is given an opportunity to choose a fixed or moving target by its celestial coordinates, orbital elements, from a list of well-known object names (including asteroids and comets), or by clicking an interactive sky chart. Once the target object is chosen, the user can define custom constraints on its visibility, airmass, Sun elevation, and separation from the Moon, enable custom offset and dithering, choose the target telescope(s) and desired bandpasses for the observation. Finally, the user is presented with a page where they specify the detailed exposure schedule, including the sequence of filters, exposure lengths, delays and repeat count, as well as time constraints. Since Skynet is a multi-user network with load balancing that attempts to share each telescope's time between different telescope owners and users on a fair basis, there's no guarantee that a long cadence will go through uninterrupted on any given telescope; for certain observations that should run without interruption, there's the target-of-opportunity (TOO) capability, and this page offers the user the ability 
Optical Observing | Observation 5043922

\begin{tabular}{|c|c|c|c|c|c|c|}
\hline \multicolumn{4}{|c|}{ Exposures } & \multicolumn{2}{|r|}{ 国 回 } & 3 of 3 image(s) taken \\
\hline & ID & Length & Telescope & Filter & Time Taken & Status \\
\hline 0 & 28800815 & $90.0 \mathrm{~s}$ & OAUJ-CDK500 & R & May 02, $202021: 20: 19$ & 回回 \\
\hline 1 & 28800816 & $90.0 \mathrm{~s}$ & OAUJ-CDK500 & $\mathrm{R}$ & May 02, 2020 21:21:53 & 回回 国 \\
\hline 2 & 28800817 & $90.0 \mathrm{~s}$ & OAUJ-CDK500 & R & May 02, 2020 21:23:26 & 国只国 \\
\hline
\end{tabular}

\begin{tabular}{|c|c|c|c|c|c|c|c|}
\hline $4 \pi$ & Type & Time Taken & t) Length & IT Filter & $\downarrow \tau$ & Binning & $\Downarrow 1$ \\
\hline 533559 & bias & April 30, 2020 - 04:58:17 & & & & 1 & 回回 \\
\hline 533595 & dark & April 30, 2020 - 05:52:46 & $80 \mathrm{~s}$ & & & 1 & 四国 \\
\hline 533689 & flat & May 4, 2020 - 02:41:51 & & $\mathrm{R}$ & & 1 & 回国 \\
\hline
\end{tabular}

Target visibility over next 24-hours when sun is below -18 degrees

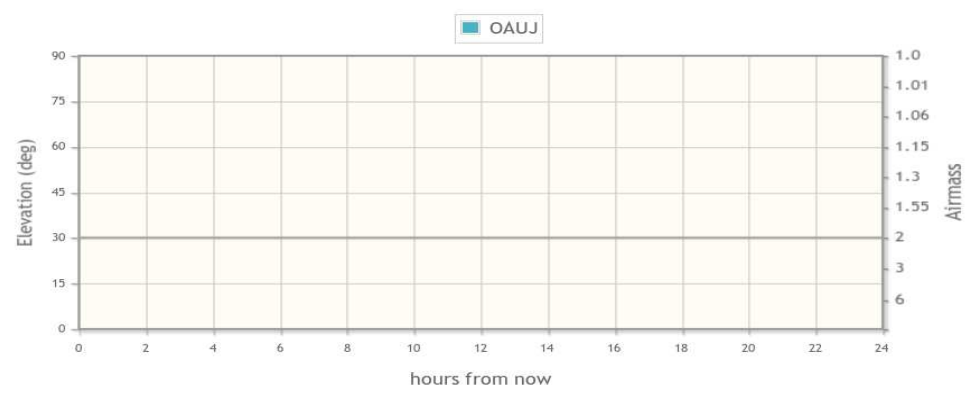

My Observations 5043922 View

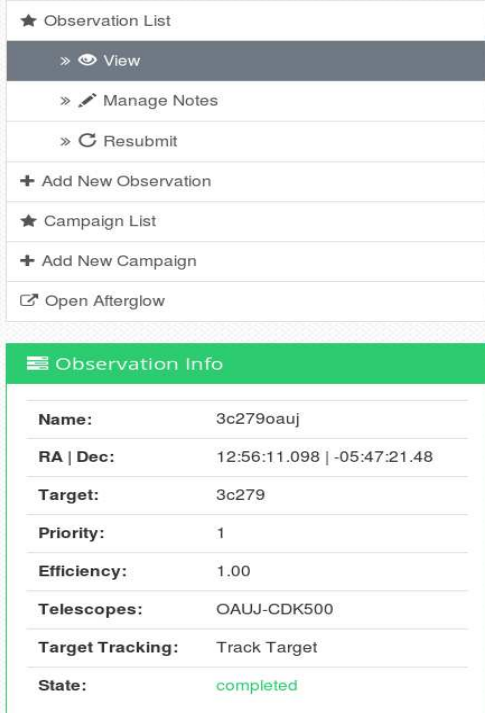

क settings

Reduction:

B/W Inversion:

REDUCED -

NOT INVERTED -

Scale Preset:

Fig. 3. Viewing and downloading observations. Both calibrated and raw images are available for download along with calibration images.

to flag the observation as TOO if they have been granted this privilege. There's also a capability to batch-upload a set of observations described in the standard way in a text file, one observation per line. This works for quickly submitting a large set of basic observations prepared in an automated way in a spreadsheet or by a user's script; however, Skynet API (see below) is much more flexible and powerful regarding this purpose.

observation as a whole in a ZIP archive or as individual FITS files, either raw or dark- and flatcorrected, plus the associated master bias, dark, and flat images (see Fig. 1.2). Under the hood, the images are uploaded from the telescope control computer to Skynet servers at UNC in the real time, archived in the database, undergo the world coordinate system (WCS) calibration, and are available for download shortly (typically seconds or fractions of a minute) after exposure is finished, depending on the image size, telescope to UNC link speed, and server load. Once the observation is completed, its owner is notified via email and/or text message.
Apart from the web UI, Skynet has a RESTful (https://restfulapi.net/) web application programming interface (API), which allows advanced users to programmatically control Skynet by means of sending hypertext transfer protocol (HTTP) requests. The API provides all capabilities available in the UI, including submitting and monitoring optical and radio observations, downloading data, and getting the current telescope state and parameters but with a much greater degree of flexibility and in a way suitable for automating complex or routine tasks, like submitting repeated observation every night or dynamically rearranging individual exposures and changing their parameters on the fly. In particular, the new Skynet framework for responding to alerts (like GRBs or LIGO/Virgo events), currently under development, is based on the API and is meant to flexibly react to such events depending on their parameters and the predefined pattern set up by Skynet users or administrators.

Skynet time sharing is based on the concept of credits - units of equivalent exposure time. One 
credit is equal to one second exposure on a standard 16-inch telescope, the role played by PROMPT-5 at CTIO. Each registered Skynet user has one or more time accounts. Each one of them provides access to a limited set of telescopes and contains a certain amount of credits deposited by a telescope owner, a group or collaboration administrator, or any other Skynet entity. Bigger entities - groups and collaborations - may have their own time accounts that they may use to deposit credits on their members' time accounts. To submit an observation, the user must have enough credits, which are immediately deducted upon submitting the observation; if the user canceled it before completion, unused credits are returned back to their time account. The same amount of credits pays different exposure length on different telescopes, depending on their efficiency. For instance, 60 credits that pay 60 seconds exposure time on PROMPT-5 (efficiency $=1$ ) would pay only 30 seconds on a telescope with efficiency $=2$. If the user wants a 60 -second exposure on such a telescope, they will pay twice the amount they would pay for PROMPT-5. Depending on the user's own preference and the goal of observation - either get the given exposure length on the given telescope or achieve the given signal-to-noise ratio (SNR) for the target on any telescope - the user may choose to submit the observation to individual telescopes separately, assuming their actual efficiencies, or to submit it to all telescopes at once assuming standard efficiency. In the first case, the corresponding amount of credits will be deducted, depending on each telescope's efficiency; in the second case, the same amount of credits will go towards each telescope, but the actual exposure length will be scaled according to the telescope's efficiency. Telescope efficiencies are calculated based on each telescope's actual performance from the images obtained over an extended period of time; they may change when the telescope gets a new camera or slowly evolve as the camera accumulates dust or its sensor degrades.

One of the most important advantages of Skynet - as any global network of telescopes - is its worldwide geographic coverage. Having telescopes in Northern and Southern hemispheres leads to the availability of targets in any part of the sky. The distribution across longitudes allows one to facilitate almost uninterrupted observations of a certain target. Having multiple sites in the same geographic regions - USA/Canada and Australia - may help to mitigate the consequences of bad weather by providing a certain degree of redundancy and provide a higher temporal resolution when observing the same

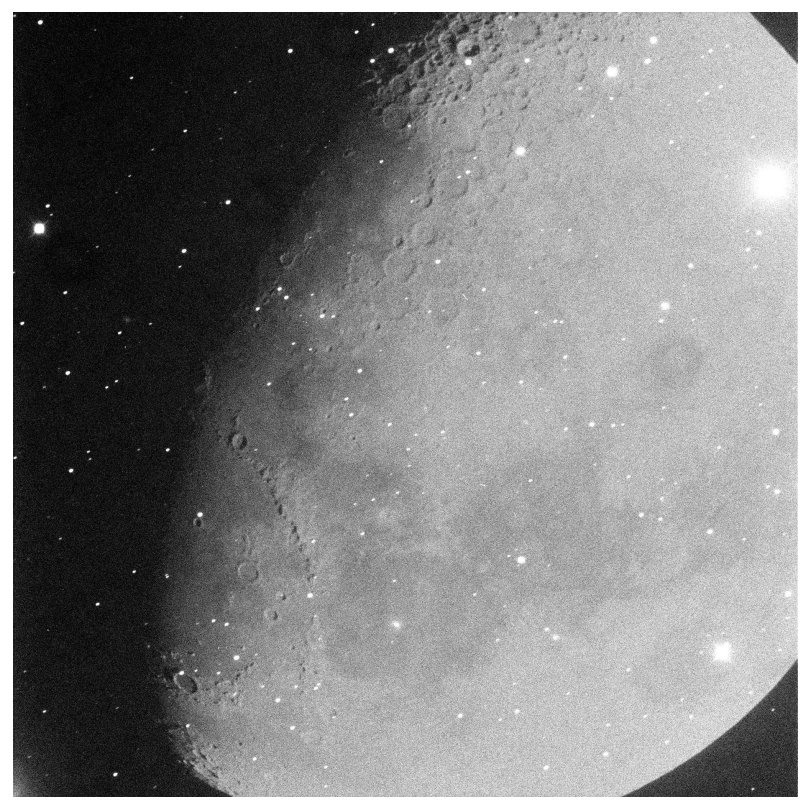

Fig. 4. An image showing one of the hardware-related issues with Skynet. An image of the Moon (still visible in the frame due to CCD memory effect) was taken right before observations of the OJ 287 blazar.

object by multiple telescopes at once. The diversity of telescopes also provides the opportunity to choose those that better suite the needs of a particular observation.

Skynet has an advanced yet easy to use web UI for submitting, watching, and downloading observations, with the capability to watch them in nearrealtime, with automatic calibration if needed. The user still doesn't have to stay awake while the observation is in progress: they may submit it and forget until it's completed, which they are immediately notified of. Beyond that, Skynet has an even more flexible means to control the observation process - the web API. Both the UI and the API are backed by a relational database of over 25 million images and metadata collected by Skynet over 15 years of its operation - a potential source for data mining. Skynet is also tightly integrated with Afterglow Access, an online astronomical image analysis tool currently under development by the Skynet team.

However, Skynet has also its pitfalls that a prospective user should be aware of. The first group of issues originates from the nature of Skynet as a multi-user network with time sharing. As a result, depending on the actual telescope load, a long run may not go uninterrupted unless submitted as a TOO, which requires some privileges. Apart from that, a recently added opportunity for the user to choose a non-default binning leads to a lack of flats 
for less frequently used binnings on some telescope. The current automatic flat scheduling algorithm is not efficient enough to take flats for all possible binnings and filters frequently enough; as a result, master flats are sometimes outdated.

The second group of problems comes from the usual hardware-related issues, like bad focus, inaccurate pointing, filter wheel failures, and CCD ghosting for cameras without RBI (residual bulk image) flooding (see Fig. 4). Sometimes, the weather sensor thresholds are improperly chosen, and as a result images are taken in poor conditions.

Most of these issues are, however, being constantly fixed by the Skynet operations staff at UNC or, on their request, by the local engineering staff at the observatories.

In the next section, we present some results obtained using Skynet observational facilities.

\section{PHOTOMETRIC MONITORING OF SELECTED BLAZARS}

We selected a sample of quasars and blazars for regular long-term monitoring of their brightness in the optical band with Skynet telescopes. Usually, the cadence of measurements is a few days, but we collect data more frequently if a target exhibits fast activity (e. g. flaring in blazars). Taking advantage of the location of the telescopes across four continents, we can increase the frequency of observations and measure a target twice a day. We take several images in the R filter, for which the efficiency of CCD detectors is the highest, and thus there is a higher chance to get more flat field images for calibration. This way we can immediately spot any interesting features in the light curves of the targets, like the beginning of flares or deep fading, and then follow them subsequently by a subset of Skynet telescopes with a higher temporal resolution. In order to investigate the variability properties of a source on a timescale of a few weeks to few months, a long-term light curve is required. Here we report the observations of two objects OJ 49 and 3C 279 covering a single observing season.

OJ $49\left(z=0.173683, \quad \mathrm{RA}=08^{\mathrm{h}} 31^{\mathrm{m}} 48.88^{\mathrm{s}}\right.$, Dec $\left.=+04^{\circ} 29^{\prime} 39.086^{\prime \prime}\right)$ is classified as a BL Lacertae object. The radio (VLA) image of the source at 20 cm (Antonucci, \& Ulvestad 1985) shows a sharply curved extended jet, whereas in a $43 \mathrm{GHz}$ polarized intensity VLBA image, the object displays a highly polarized jet, extending about 0.6 mas from the core (Lister et al. 1998). VLBA images at $22 \mathrm{GHz}$ revealed a prominent jet ejecting knotty components at a superluminal speed (Jorstad et al. 2001). During VLBI Space Observatory Program mission, the
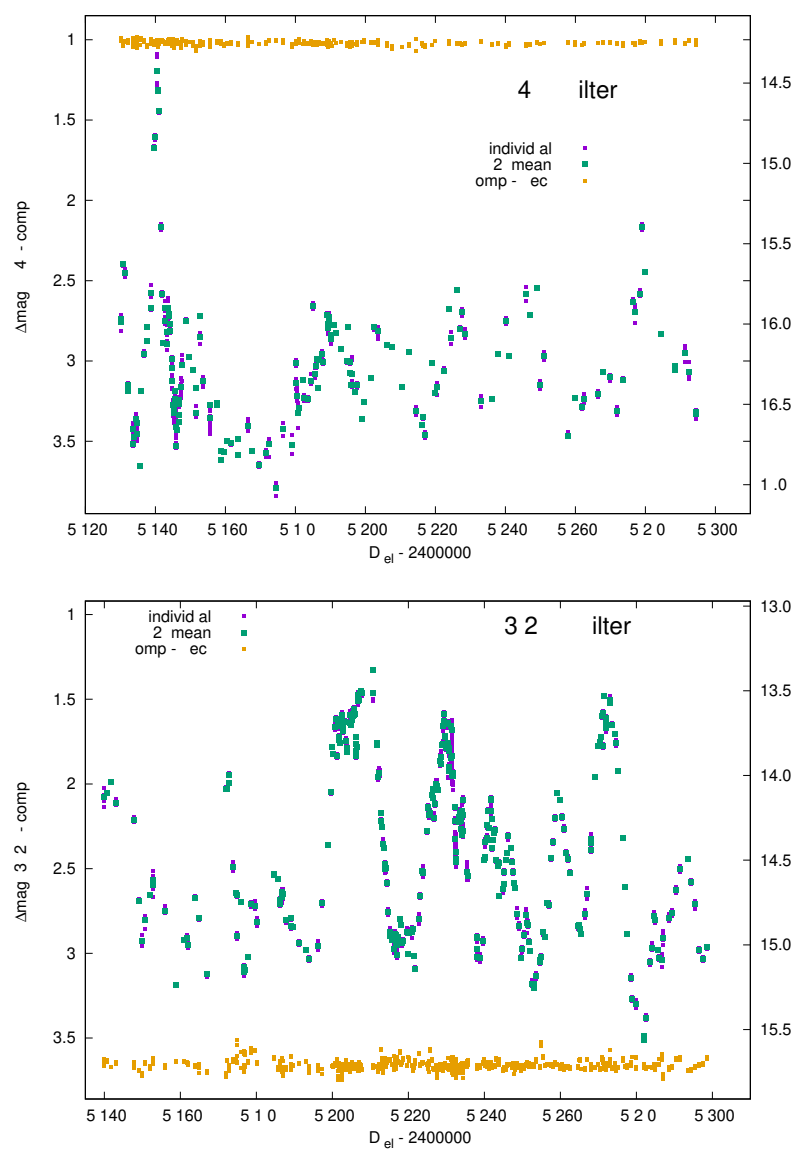

Fig. 5. Light curves of blazars OJ 49 (top) and 3C 279 (bottom). All data shown here were taken with Skynet telescopes.

core size of the $5 \mathrm{GHz}$ radio emission was estimated to be 0.5 mas (Dodson et al. 2008). In the optical and near-IR bands, the blazar exhibits strong intraday variability of polarization and flux (see Sitko et al. 1985; Smith et al. 1987).

Blazar 3C $279\left(z=0.536, R A=12^{\mathrm{h}} 56^{\mathrm{m}} 11.1665^{\mathrm{s}}\right.$, Dec $\left.=-05^{\circ} 47^{\prime} 21.523^{\prime \prime}\right)$ is a powerful flat spectrum radio quasar, emitting prominently in hard X-ray and gamma rays. The source, which is highly variable across the entire electromagnetic spectrum (see Hayashida et al. 2015; Paliya et al. 2016, and references therein), is one of few objects detected above $100 \mathrm{GeV}$ (MAGIC Collaboration et al. 2008). Observations of its structure in radio revealed a compact, milliarcsecond-scale radio core, ejecting knots with a bulk Lorentz factor $\Gamma=15.5 \pm 2.5$ along the direction with an angle of $\theta_{\text {obs }}=2.1 \pm 1.1^{\circ}$ with respect to the line of sight (Jorstad et al. 2005, 2004). During the Whole Earth Blazar Telescope (WEBT) campaigns, the source flux in the optical was reported to decay exponentially on a timescale of $\sim 10 \mathrm{~d}$ (Böttcher et 
al. 2007). Also Larionov et al. (2008), based on data from another WEBT campaign obtained in 20062007 , found a slower ( $\sim 100 \mathrm{~d}$ timescale) but more prominent $(\sim 3 \mathrm{mag})$ flux decrease in the optical and near-IR bands. Bhatta, Mohorian, \& Bilinsky (2018) discussed the flux and spectral variability properties of the source in hard X-ray over intraday timescales. Using the NuSTAR observations, they found that the source flux exhibited a large amplitude variability, nearly doubling on a timescale of a few hours. In addition, the hard X-ray spectrum could be characterized by a broken power-law and log-parabolic spectral models, suggesting the presence of a spectral break within the $3-79 \mathrm{KeV}$ energy range. More recently, Agarwal et al. (2019) detected multi-band optical variability of the source and found high amplitude variability on a timescale of a few months and a mild bluer-when-brighter trend on somewhat shorter timescales.

The objects' near-equator declinations make their observations from both hemispheres possible; therefore, a cadence of two observations per day can be achieved with Skynet telescopes. In Fig. 5, we show the light curves taken in the 2018/2019 observing seasons. We recorded a spectacular flare at the beginning of OJ 49 run that lasted about 10 days, when the brightness of the blazar in the $\mathrm{R}$ filter increased by $2.3 \mathrm{mag}$, from 16.5 to about 14.2 . Within a few days, OJ 49 returned to the pre-outburst level, exhibiting variations with lower amplitude over the next 7 months. In 2018, we performed a monitoring of 3C 279 in the R band during nearly 7 months. The target flux variability was extremely strong: its brightness was going up and down by over 1.5 mag.

\subsection{Variability Analysis}

In order to quantify the observed variability of the sources, we estimated the variability amplitude of the sources for the observation period. It provides a measure of the peak-to-peak flux variations and can be written as

$$
\mathrm{VA}=\sqrt{\left(A_{\max }-A_{\min }\right)^{2}-2 \sigma^{2}},
$$

where $A_{\max }, A_{\min }$, and $\sigma$ are the maximum, minimum, and mean of the magnitude errors in the light curves, respectively (see Heidt, \& Wagner 1996). Since this kind of measure takes only flux extrema into consideration and therefore does not account for all observed data, a measure of the average variability can be provided as the fractional variability (FV) parameter (proposed by Vaughan et al. 2003), expressed as:
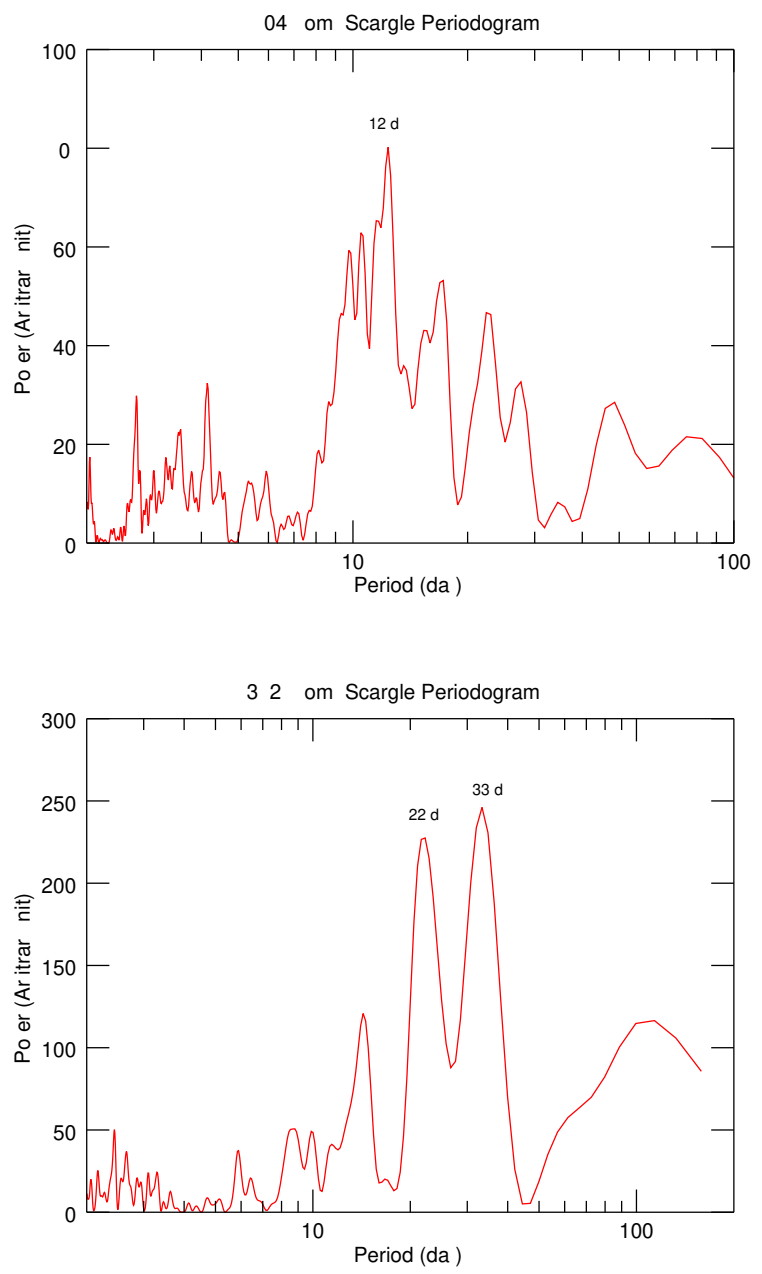

Fig. 6. The Lomb-Scargle periodograms of OJ 49 (upper panel) and 3C 279 (bottom panel) showing possible periodicities.

$$
F_{v a r}=\sqrt{\frac{S^{2}-\left\langle\sigma_{e r r}^{2}\right\rangle}{\langle F\rangle^{2}}},
$$

where, for a time series with a mean flux $\langle F\rangle, S^{2}$ and $\left\langle\sigma_{e r r}^{2}\right\rangle$ represent variance and mean squared uncertainties, respectively. The error in the fractional variability can be given as

$\sigma_{F_{\text {var }}}=\sqrt{F_{\text {var }}^{2}+\sqrt{\frac{2}{N} \frac{\left\langle\sigma_{\text {err }}^{2}\right\rangle^{2}}{\langle F\rangle^{4}}+\frac{4}{N} \frac{\left\langle\sigma_{\text {err }}^{2}\right\rangle}{\langle F\rangle^{2}} F_{v a r}^{2}}}-F_{\text {var }}$

(see Bhatta \& Webb 2018).

The variability timescale can be taken as the e- 
folding timescale of flux change, given by

$$
\tau_{\text {var }}=\left|\frac{\Delta t}{\Delta \ln F}\right|,
$$

(Burbidge et al. 1974, see also Bhatta, Mohorian, \& Bilinsky 2018), where $\Delta t$ is the time interval corresponding to the change in natural logarithm of flux measurements.

\subsection{Periodicity Analysis}

Quasi-periodic oscillations (QPOs) in blazars with characteristic timescales of a few years have been frequently reported (Bhatta et al. 2016c) (see also Zola et al. 2016). However, quite a few blazar QPOs on timescales of a few days or weeks were observed. We searched for the possible periodic flux modulations in optical using the LombScargle method (Lomb 1976; Scargle 1982). The method modifies the conventional discrete Fourier periodogram in such a way that the least-squares fitting of sine waves to the data in the form $X_{f}(t)=$ $A \cos \omega t+B \sin \omega t$ is minimized. The periodogram is given by

$$
\begin{aligned}
P= & \frac{1}{2}\left\{\frac{\left[\sum_{i} x_{i} \cos \omega\left(t_{i}-\tau\right)\right]^{2}}{\sum_{i} \cos ^{2} \omega\left(t_{i}-\tau\right)}+\right. \\
& \left.\frac{\left[\sum_{i} x_{i} \sin \omega\left(t_{i}-\tau\right)\right]^{2}}{\sum_{i} \sin ^{2} \omega\left(t_{i}-\tau\right)}\right\},
\end{aligned}
$$

where $\tau$ is given by

$$
\begin{gathered}
\tan (2 \omega \tau)=\sum_{i} \sin \omega t_{i} / \sum_{i} \cos \omega t_{i} . \\
\text { 2.3. Results } \\
\text { OJ } 49
\end{gathered}
$$

We observed a high optical variability estimated as $\mathrm{VA}=2.75 \mathrm{mag}$ and an average variability with $\mathrm{FV}=66.23 \pm 0.24 \%$. The variability timescale equivalent to e-folding timescales was measured to be $38.24 \pm 11.80$ minutes. As revealed by Lomb-Scargle periodogram, a strong periodic signal of $\sim 12$ days may be present in the OJ 49 light curve.

$$
3 C 279
$$

Similar to OJ 49, we found the source to be highly variable with VA of $2.78 \mathrm{mag}$ and $\mathrm{FV}$ of $46.16 \pm$ $0.50 \%$. Measured variability timescale was $11.73 \pm$ 7.80 minutes, while the periodicity analysis indicated the presence of two possible periods of 22 and 33 days. Interestingly, the two periodicities that appear to be at the 2:3 ratio resemble the ones claimed to be present in X-ray binaries (e.g. Abramowicz et al. 2003).

\section{CONCLUSIONS}

In this paper, we focused on observations performed with Skynet optical telescopes; however, radio data could be collected as well with the Green Bank 20m (GBT-20) antenna, also a member of the network. GBT-20 is available via the same user interface and API to registered Skynet users. The principles of its operation and a detailed description of the single-dish mapping algorithm to be integrated into Skynet image processing software were given by Martin et al. (2019). With this setup, a simultaneous optical and radio data collection can be facilitated.

We argue that the software controlling optical Skynet telescopes is convenient and easy to use both by professional astronomers and untrained users, e. g. students. An intuitive web user interface and a flexible API provide a way to schedule either Basic or arbitrarily complex observations, taking into account the diversity of Skynet telescope hardware. From the perspective of a regular user doing non-high priority observations, the Skynet Telescope Network turned out to be most suitable for observations requiring long term monitoring and short runs taken with a cadence of one to few nights. For such programs, the network could provide data equivalent to what could be obtained in a multi-site campaign, but with just a single person (or a very few persons) required to schedule observations, download the data, and inspect the results to avoid/minimize the possible pitfalls described in this work.

We statistically analyzed the light curves of two blazars: OJ 49 and 3C 279. Their light curves were collected with Skynet telescopes and cover one observing season. Location of the telescopes and their redundancy at the same longitudes allowed for obtaining dense light curves. Both targets turned out to be highly variable on a timespan of about seven months, with $\mathrm{VA}=2.8$ magnitudes and FV between 66 and $46 \%$. For OJ 49, we recorded one prominent flare; several repeating outbursts were noticed in the 3C 279 light curve, although with a lower amplitude. In total, we are currently monitoring the brightness of 5 blazars with Skynet, and the results of the analysis of the light curves of the entire sample will be published elsewhere (Bhatta et al. 2020). The efficiency of the Skynet Robotic Telescope Network was demonstrated on two other targets: Ark 120 and OJ 287. The former, a nearby Seyfert-type galaxy, was a target of a simultaneous SWIFT and multisite optical campaign. It turned out that Skynet telescopes provided more than 70 percent of observations (Lobban 2020) but involved just a single observer scheduling jobs and another person reducing 
the images. Since 2015, OJ 287 has been regularly monitored with Skynet, and a very dense light curve of this blazar allowed to pinpoint a deep fading event that occurred in 2017. Followup observations with GTC resulted in the detection of the blazar's host galaxy (Nilsson et al. 2020).

Acknowledgements: The authors acknowledge support from the following grants and institutions: NCN grant No. 2018/29/B/ST9/01793 (SZ).

\section{REFERENCES}

Abramowicz, M. A., Bulik, T., Bursa, M., et al. 2003, A\&A, 404, L21

Agarwal, A., Cellone, S. A., Andruchow, I., et al. 2019, MNRAS, 488, 4093

Antonucci, R. R. J., \& Ulvestad, J. S. 1985, ApJ, 294, 158

Bhatta, G., Mohorian M., and Bilinsky I. 2018 A\&A, 619, A93

Bhatta, G. 2018, Galaxies, 6, 136

Bhatta, G., \& Webb, J. 2018, Galaxies, 6, 2

Bhatta, G., Zola S., Stawarz, Ł., et al. 2016c, ApJ, 832, 47

Bhatta, G., et al. 2020, in preparation

Böttcher, M., Basu, S., Joshi, M., et al. 2007, ApJ, 670, 968

Burbidge, G. R., Jones, T. W., \& Odell, S. L. 1974, ApJ, 193, 43

Dodson, R., Fomalont, E. B., Wiik, K., et al. 2008, ApJS, 175,314
Hayashida, M., Nalewajko, K., Madejski, G. M., et al. 2015, ApJ, 807, 79

Heidt, J., \& Wagner, S. J. 1996, A\&A, 305, 42

Jorstad, S. G., Marscher, A. P., Mattox, J. R., et al. 2001, ApJS, 134, 181

Jorstad, S. G., Marscher, A. P., Lister, M. L., et al. 2004, AJ, 127, 3115

Jorstad, S. G., Marscher, A. P., Lister, M. L., et al. 2005, AJ, 130, 1418

Larionov, V. M., Jorstad, S. G., Marscher, A. P., et al. 2008, A\&A, 492, 389

Lister, M. L., Marscher, A. P., \& Gear, W. K. 1998, ApJ, 504,702

Lobban, A. P., Zola, S., Pajdosz-Smierciak, U. et al. 2020, MNRAS, submitted

Lomb, N. R. 1976, Ap\& SS, 39, 447

MAGIC Collaboration, Albert, J., Aliu, E., et al. 2008, Science, 320, 1752

Martin, J. R., Reichart, D. E., Dutton, D. A. et al. 2019, ApJS, 240, 12

Nilsson, K., Kotilainen, M., Valtonen, M. al. 2020, ApJ, submitted

Paliya, V. S., Diltz, C., Böttcher, M., et al. 2016, ApJ, 817,61

Scargle, J. D. 1982, ApJ, 263, 835

Sitko, M. L., Schmidt, G. D., \& Stein, W. A. 1985, ApJS, 59, 323

Smith, P. S., Balonek, T. J., Elston, R., et al. 1987, ApJS, 64,459

Vaughan, S., Edelson, R., Warwick, R. S., \& Uttley, P., 2003, MNRAS, 345, 1271

Zola, S., Valtonen, M., Bhatta, G., et al. 2016, Galaxies, 4, 41 